\title{
IV. Die konduktometrische Bestimmung von Kohlensäure und ihren Salzen.
}

\author{
Von J. M. KoLthofF. \\ Mit 5 Figuren im Text.
}

Obgleich in einem vorigen Aufsatz schon eine allgemeine Übersicht gegeben wurde von der Anwendung von konduktometrischen Titrationen in der Neutralisationsanalyse, werden Kohlensäure und Phosphorsäure wegen ihrer großen Bedeutung in den verschiedenen Zweigen der Chemie, noch absonderlich behandelt werden. Bekanntlich ist Kohlensäure eine zweibasische säure, von der sowohl die erste als die zweite Dissoziationskonstante klein ist; jedoch die erste Konstante \pm 5000 mal größer als die erste $\left(K_{1}=3 \times 10^{-7}\right.$, $\left.K_{2}=6 \times 10^{-11}\right)$. Da nun außerdem die Beweglichkeiten des Bikarbonat- und Karbonations genügend verschieden sind, kann man erwarten, daß Kohlensäure konduktometrisch sowohl als ein- und zweibasische Säure titrierbar ist. Man kann außerdem im voraus wissen, daß der Umschlag bei Bikarbonat nicht scharf sein wird; aber dies ist ebensowenig auf Farbeindikatoren der Fall. Wenn man nun oft farbige Flüssigkeiten hat, die Kohlensäure enthalten (farbiges Wasser; viele psychologisehe Flüssigkeiten usw.), ist es von Interesse zu wissen, wie Kohlensäure sich bei der konduktometrischen Titration verhält.

Die Ergebnisse der konduktometrischen Titration wurden mit denen verglichen, welche ich durch direkte Titration auf Phenolphthalein oder durch Titration zu Karbonat (Hinzufügung von Überschuß Lauge und Bariumsalz und Rücktitration) erhalten habe. Bekanntlich ist der relative Fehler dieser Methoden, besonders bei Anwesenheit von wenig Kohlensäure ziemlich groß. Also werden leicht Fehler von einigen Prozenten gemacht.

Als Beispiel der konduktometrischen Titration von Kohlensäure werden hier die Ergebnisse der Bestimmung von $\pm 0,0006$ mol. and \pm 0.0015 mol. $\mathrm{CO}_{2}$ gegeben. 
$100 \mathrm{~cm} \pm 0,006 \mathrm{~mol}$. Kohlensäure

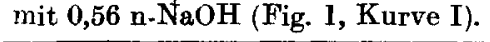

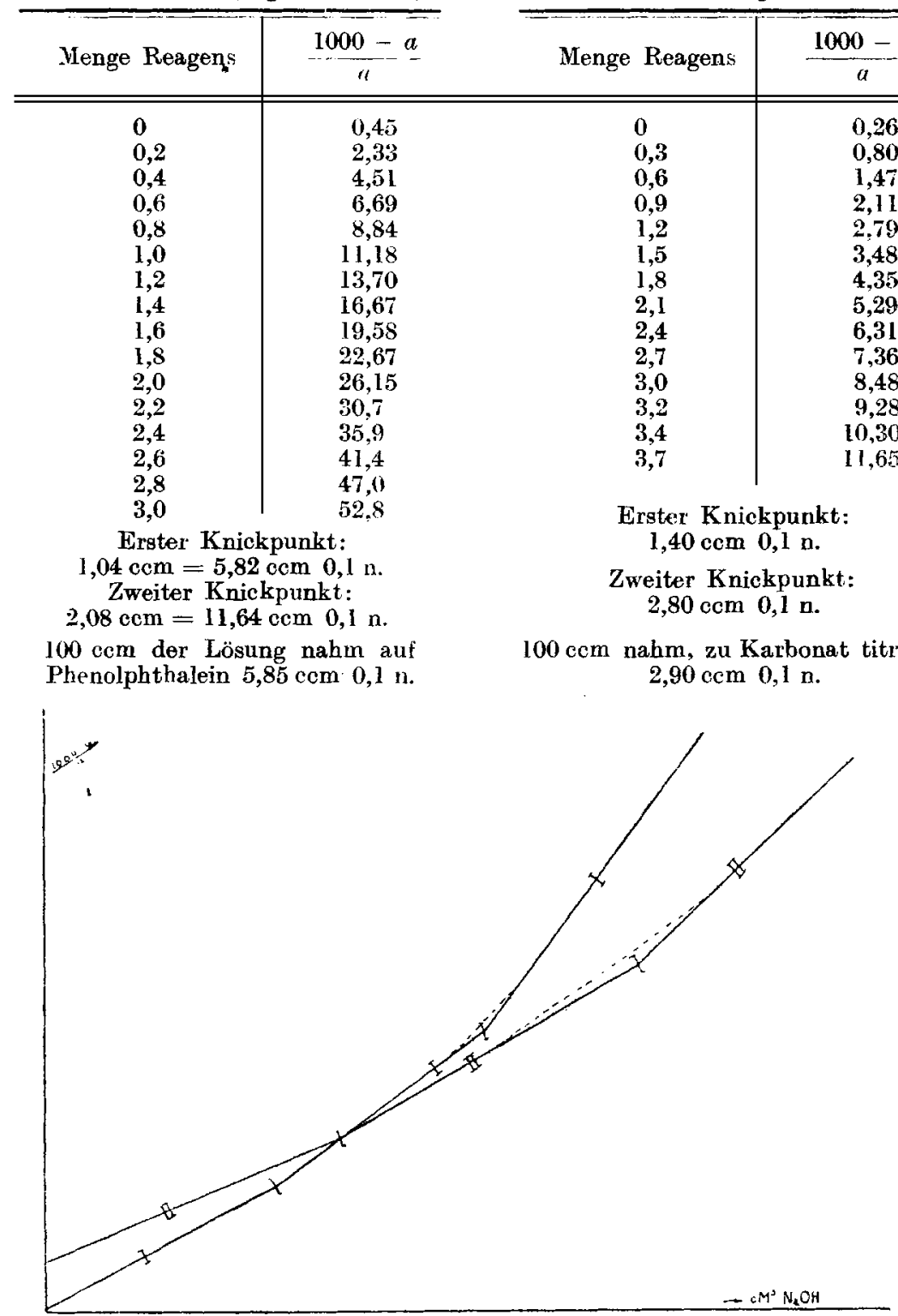

$100 \mathrm{ccm} \pm 0,0015 \mathrm{~mol}$. Kohlensäure mit 0,1 n- $\mathrm{NaOH}$ (Fig. 1, Kurve II).

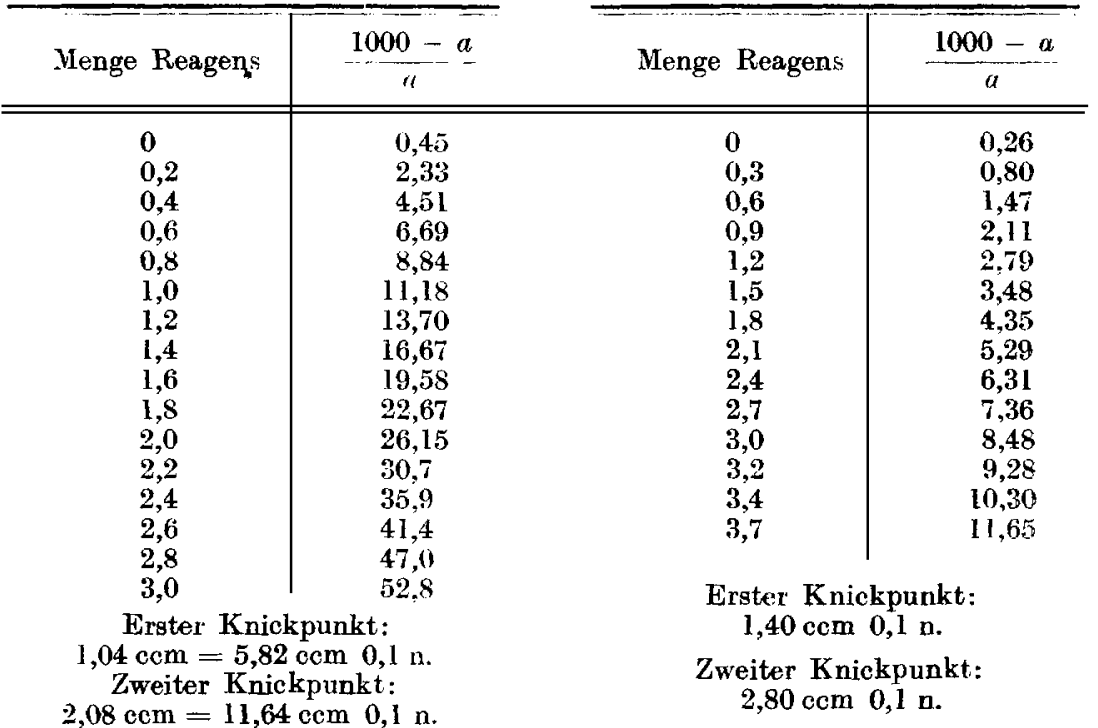

$100 \mathrm{com}$ der Lösung nahm auf Phenolphthalein 5,85 $\mathrm{cm} 0,1 \mathrm{n}$. mm, zu Karbonat titriert, $2,90 \mathrm{~cm} 0,1 \mathrm{n}$.

Fig. 1.

Auch wurden titriert $0,004,0,003$ und 0,02 mol. Kohlensäurelösungen; die Ergebnișse stimmten genügend überein mit denen, welche durch Titration auf Indikatoren erhalten wurden. 
Ein Vorteil der konduktometrischen Titration ist, daB man nach der Hinzufügung von Lauge nicht zu warten braucht, bis das Gleichgewicht sich genau eingestellt hat. Wenn man eine Kohlensäurelösung auf Phenolphthalein titriert, läuft die Farbe jedesmal zurück, weil das Gleichgewicht

$$
\mathrm{CO}_{2}+\mathrm{OH}^{\prime} \rightleftharpoons \mathrm{HCO}_{3}
$$

sich langsam einstellt. Man soll zur Erreichung des guten Endpunktes so lange titrieren, bis die rosa Farbe nach 10-15 Minuten Stehen (abhängig von der Temperatur) nicht mehr zurückläuft. Es ist leicht einzusehen, $d a B$ man bei einer konduktometrisehen Titration nicht so lange zu warten braucht, bis das Gleichgewicht sich genau eingestellt hat.

Ein Nachteil der konduktometrischen Titration dagegen ist, daß die Knicke nicht scharf sind. Wie zu erwarten ist, nimmt die Leitfähigkeit vom Anfange der Titration zu, nach der Bildung von Bikarbonat ist die Zunahme größer, und sie nimmt schließlich nach der Umwandlung in Karbonat stärker zu. Je verdünnter nun die Kohlensäurelösung ist, um so stärker ist die Hydrolyse des Karbonats und um so unschärfor ist der Knick beim Karbonat (vgl. Kurve I und II, Fig. 1). Die Ergebnisse der konduktometrischen Titrationen sind also einigermaßen ungewiß.

Ich habe nun geprüft, was der Einfluß der Anwesenheit von Calciumsalz ist. Letzteres kommt sowohl in allen Trinkwassern als in physiologischen Flüssigkeiten vor. Ess stellte sich nun heraus, $\mathrm{da} B$, wenn genügend Calcium anwesend ist, die Knicke viel schärfer werden. Nachdem die Kohlensäure in Bikarbonat verwandelt ist, fängt bei fortgesetzter Hinzufügung von Lauge das Calciumkarbonat an zu präzipitieren. Man soll dann jedoch jedesmal nach der Hinzufügung einer bestimmten Menge Lauge eine bis zwei Minuten warten, bevor die Leitfähigkeit konstant ist. Wenn die zu titrierende Lösung sehr wenig Kohlensäure enthält und der Calciumgehalt nicht zu groß ist, dauert es lange, bis das Calciumkarbonat anfängt zu präzipitieren. In diesem Falle kann man besser weiter titrieren und ist es besser, nicht zu warten, bis der Karbonatniederschlag anfängt, sich zu bilden. Durch die Anwesenheit des Calciumsalzes wird die Hydrolyse des Karbonats zurückgedrängt, so daß der Knick viel schärfer ist. Von den verschiedenen Titrationen, die ich gemacht habe, lasse ich unten zwei Beispiele folgen. 
$100 \mathrm{ccm}+0,005 \mathrm{~mol}$. Kohlensäure und $20 \mathrm{mg}$ Calcium als Chlorid mit 0,56 n-NaOH (Fig. 2, Kurve I).

\begin{tabular}{c|c}
\hline \hline $\begin{array}{c}\text { Zugefügte } \\
\text { ccm Reagens }\end{array}$ & $1000-a$ \\
\hline 0 & $a$, \\
0,2 & 27,6 \\
0,4 & 39,5 \\
0,6 & 33,5 \\
0,8 & 35,3 \\
1,0 & 36,5 \\
1,2 & 36,6 \\
1,4 & 36,6 \\
1,6 & 36,7 \\
1,8 & 36,9 \\
$\mathbf{2 , 0}$ & 38,5 \\
2,2 & 43,0 \\
2,4 & 48,2 \\
2,6 & 53,8 \\
2,8 & $\mathbf{5 9 , 5}$
\end{tabular}

Erst.Knickp. : $0,94 \mathrm{ccm}=5,26 \mathrm{ccm} 0,1 \mathrm{n}$. Zweit. , $1,99, \quad=11,0,0,1 \mathrm{n}$. $100 \mathrm{ccm}$ nahmen auf Phenolphtalein $5,40 \mathrm{ccm} 0,1 \mathrm{n}$-Lauge.
$100 \mathrm{ccm} \pm 0,001 \mathrm{~mol}$. Kohlensäure und $20 \mathrm{mg}$ Calcium als Chlorid mit 0,1 n-Lauge (Fig. 2, Kurve II).

\begin{tabular}{c|c}
\hline $\begin{array}{c}\text { Zugefügte } \\
\text { com Reagens }\end{array}$ & $1000-a$ \\
\hline \hline 0 & 31,65 \\
0,3 & 32,1 \\
0,6 & 32,45 \\
0,9 & 32,8 \\
1,2 & 32,15 \\
1,5 & 33,3 \\
1,8 & 33,25 \\
2,0 & 33,20 \\
2,2 & 33,14 \\
2,4 & 33,2 \\
2,6 & 33,4 \\
3,0 & 34,7 \\
3,3 & 36,0 \\
3,6 & 37,4 \\
3,8 & 38,2
\end{tabular}

Erster Knickpunkt: $1,28 \mathrm{ccm} 0,1 \mathrm{n}$. Zweiter ", $\quad 2,63 \quad, \quad 0,1$ n. $100 \mathrm{ccm}$ nahmen auf Phenolphthalein $1,32 \mathrm{ccm} 0,1 \mathrm{n}$-Lauge.

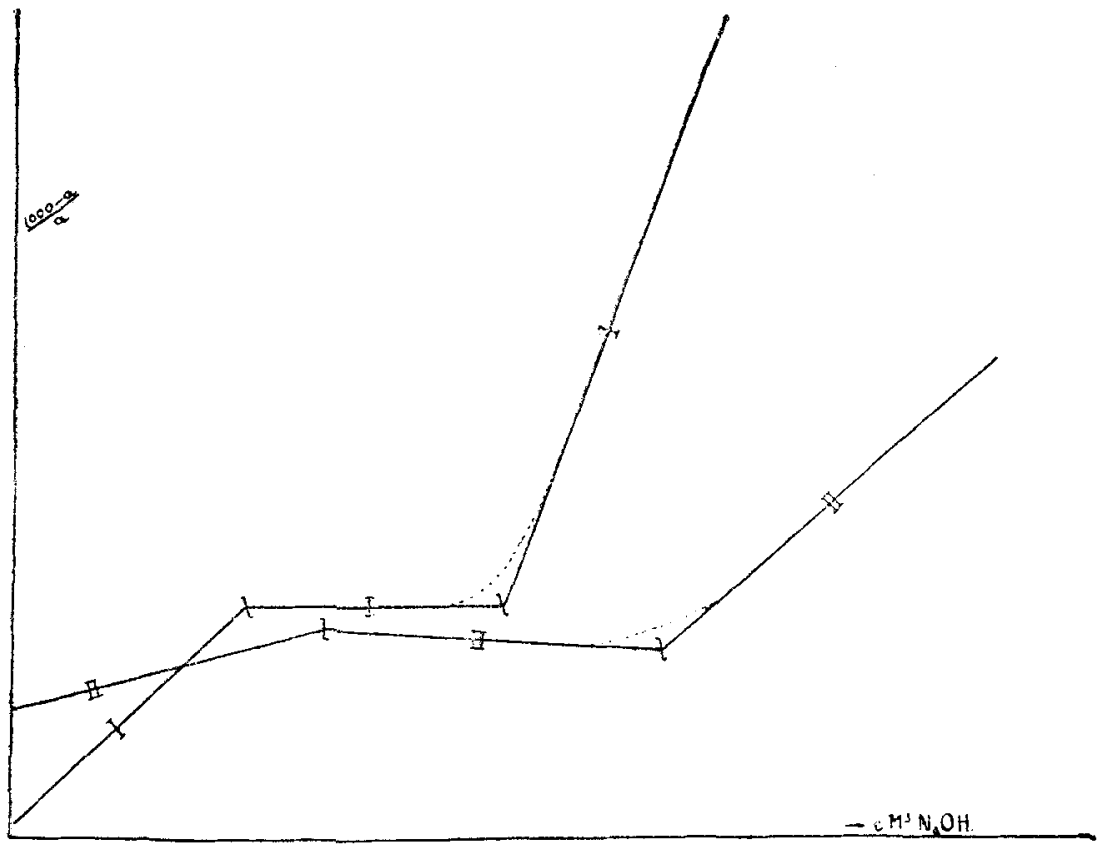

Fig. 2.

Wenn zu $100 \mathrm{ccm}$ der $0,005 \mathrm{~mol}$. Kohlensäurelösung nur $10 \mathrm{mg}$ Calcium als Chlorid gefügt wurde, wurden unregelmäßige Ergebnisse 
erhalten. Man muß also dafür sorgen, đaß ein Überschuß an Calcium anwesend ist.

Schließlich versuchte ich, Kohlensäure unter verschiedenen Umständen konduktometrisch mit Natriumkarbonat zu titrieren. Hier fand ich nur einen Knickpunkt bei Bikarbonat; der zwischen den beiden Linien gebildete Winkel ist aber so stumpf, daß man mit dieser Titration praktisch keine brauchbaren Resultate erhält.

Sodann habe ich das Verhalten von Natriumkarbonatlösungen bei der konduktometrischen Titration geprüft. Meerburg (1910 und 1911) hat schon darauf hingewiesen, daß die Verwendung der konduktometrischen Bestimmung von Bikarbonat in farbigem Wasser von Bedeutung ist. Die Graphik, welche er von der Neutralisation von Karbonat mit Säure gibt, ist aber nicht richtig.

In den folgenden Beispielen werde ich zuerst die Ergebnisse der konduktometrischen Titration von Natriumkarbonat bei verschiedenen Verdünnungen mitteilen. Die Lösungen wurden aus einem reinen Präparat bereitet, das vorher auf $360^{\circ}$ bis zu konstantem Gewicht erhitzt war; das verwendete Wasser war kohlensäurefrei. Die Bestimmungen sind bei jeder Verdünnung 3 bis 4 mal ausgeführt, die Resultate stimmten immer miteinander überein. In Fig. 3 findet man die Verdrängungslinien von $0,1-0,01 \mathrm{n}$ - und $0,0025 \mathrm{n}$-Karbonat dargestellt. Die Resultate mit 0,0001 n-Karbonat findet man außerdem in folgender Tabelle erwähnt.

$25 \mathrm{cem} 0,099 \mathrm{n}-\mathrm{Na}_{2} \mathrm{CO}_{3}(=0,0495 \mathrm{~mol}$.) mit n-HCl (Fig. 3, Kurve I).

\begin{tabular}{c|c}
\hline \hline $\begin{array}{c}\text { Zugefügte } \\
\text { ccm Reagens }\end{array}$ & $\frac{1000-a}{\alpha}$ \\
\hline \hline 0 & 13,70 \\
0,3 & 13,75 \\
0,6 & 13,92 \\
0,9 & 14,16 \\
1,2 & 14,42 \\
1,5 & 14,87 \\
1,8 & 15,32 \\
2,1 & 15,84 \\
2,4 & 16,32 \\
2,8 & 24,24 \\
3,0 & 28,9 \\
3,2 & 33,68 \\
3,4 & 38,54 \\
&
\end{tabular}

Erster Knickpunkt: $1,24 \mathrm{ccm}$ (ber. $1,245 \mathrm{ccm}$ ).

Zweiter Knickpunkt: $2,48 \mathrm{ccm}$ (ber. $2,475 \mathrm{ccm}$ ).
25 cem 0,01 n- $\mathrm{Na}_{2} \mathrm{CO}_{3}(=0,005 \mathrm{~mol}$. mit 0,1035 n-HCl (Fig. 3, Kurve II).

\begin{tabular}{c|c}
\hline \hline $\begin{array}{c}\text { Zugefügte } \\
\text { ccm Reagens }\end{array}$ & $\frac{1000-a}{a}$ \\
\hline \hline 0 & 15,90 \\
0,2 & 16,03 \\
0,4 & 16,20 \\
0,6 & 16,10 \\
0,8 & 16,05 \\
1,0 & 16,05 \\
1,2 & 16,18 \\
1,4 & 16,60 \\
1,6 & 17,03 \\
1,8 & 17,40 \\
2,0 & 17,85 \\
2,2 & 18,33 \\
2,4 & 19,16 \\
2,6 & 24,6 \\
2,8 & 30,2 \\
3,0 & 35,8 \\
3,2 & $\mathbf{4 1 , 3}$
\end{tabular}

Erst. Knickp. : 1,16ccm(ber.1,195ccm). Zweit. ," 2,385 , (ber. 2,39 ,). 
$100 \mathrm{ccm} 0,00247 \mathrm{a}-\mathrm{Na}_{2} \mathrm{CO}_{3}$ in ausgekochtem Wasser mit $0,1035 \mathrm{n} \cdot \mathrm{HCl}$ (Fig. 3, Kurve III).

\begin{tabular}{c|c}
\hline $\begin{array}{c}\text { Zugefügte } \\
\text { ccm Reagens }\end{array}$ & $\frac{1000-a}{u}$ \\
\hline \hline 0 & 6,80 \\
0,2 & 6,58 \\
0,4 & 6,42 \\
0,6 & 6,29 \\
0,8 & 6,21 \\
1,0 & 6,16 \\
1,2 & 6,18 \\
1,6 & 6,53 \\
1,8 & 6,69 \\
2,0 & 6,87 \\
2,2 & 7,06 \\
2,4 & 7,45 \\
2,6 & 9,38 \\
2,8 & 11,50 \\
3,0 & 13,70 \\
3,2 & 15,84
\end{tabular}

Erster Knickpunkt: $1,08 \mathrm{ccm}$ (ber. $1,195 \mathrm{ccm}$ ).

Zweiter Knickpunkt: $2,390 \mathrm{~cm}$ (ber. $2,390 \mathrm{ccm}$ ).
$100 \mathrm{ccm} 0,001 \mathrm{n} \cdot \mathrm{Na}_{2} \mathrm{CO}_{33}$ $(=0,0005 \mathrm{~mol}$. $)$ mit $0,1035 \mathrm{n} \cdot \mathrm{HCl}$.

\begin{tabular}{c|c}
\hline \hline $\begin{array}{c}\text { Zugefügte } \\
\text { ccm Reagens }\end{array}$ & $\frac{1000-a}{a}$ \\
\hline \hline 0 & 2,74 \\
0,1 & 2,62 \\
0,2 & 2,53 \\
0,3 & 2,45 \\
0,4 & 2,43 \\
0,5 & 2,47 \\
0,6 & 2,56 \\
0,7 & 2,65 \\
0,8 & 2,74 \\
0,9 & 2.84 \\
1,0 & 3,21 \\
1,2 & 5,20 \\
1,4 & 7,36 \\
1,6 & 9,49 \\
1,8 & 11,60
\end{tabular}

Erster Knick punkt: $0,425 \mathrm{ccm}$ (ber. $0,48 \mathrm{ccm}$ ).

Zweiter Knickpunkt : $0,983 \mathrm{ccm}$ (ber. $0,975 \mathrm{ccm}$ ).

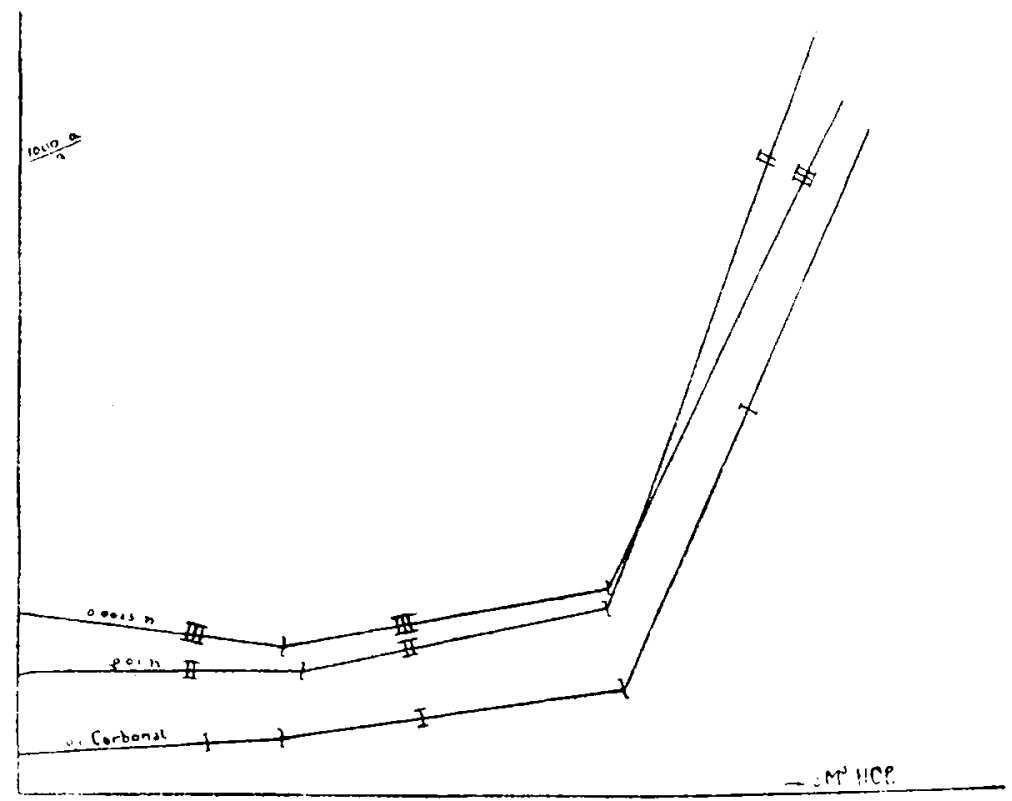

Fig. 3.

Hinsichtlich der erhaltenen Resultate ist folgendes zu bemerken: Die Richtung der Verdrängungslinie von Natriumkarbonat zu Bi- 
karbonat hängt von der Konzentration des Karbonats ab. In stärkeren Lösungen steigt die Linie ein wenig, in verdünnteren Lösungen fällt sie. Dies hängt mit dem zunehmenden Dissoziationsgrad und mit der Hydrolyse der Karbonatlösung bei steigender Verdünnung zusammen. Der Knick ist bei Bikarbonat nie scharf. Ebensowenig als mit Farbeindikatoren kann man Karbonat genau zu Bikarbonat titrieren. In stark verdünnter Karbonatlösung stört außerdem die gelöste Kohlensäure, so daß dann der Knickpunkt

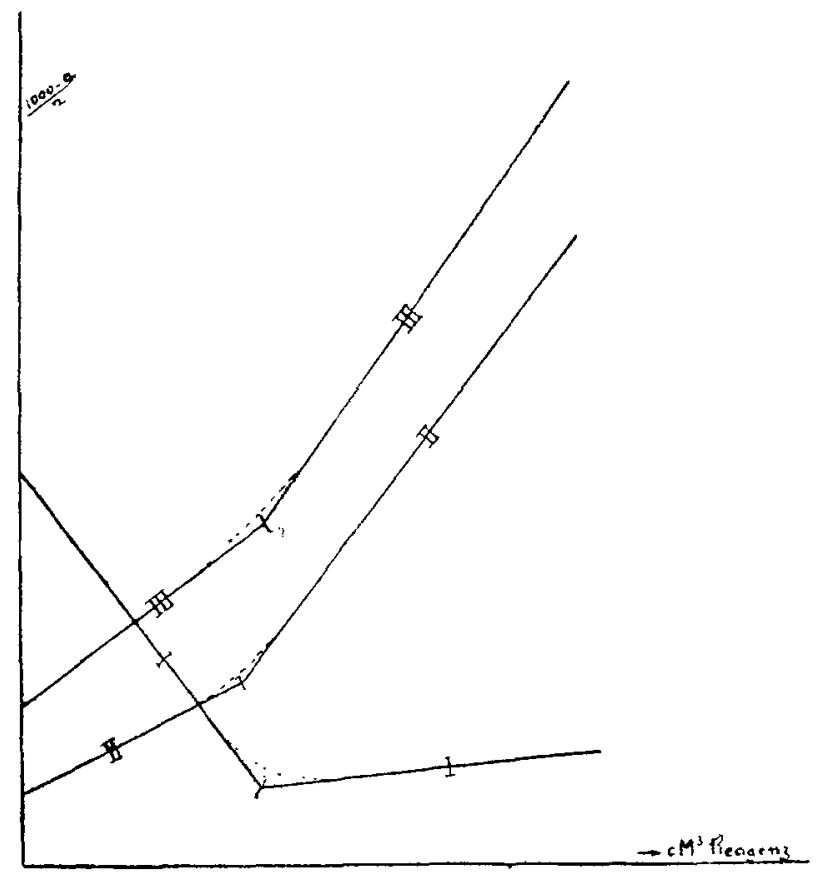

Fig. 4.

viel zu früh gefunden wird (vgl. die Titration von 0,0025 mol. Karbonat). Die Bestimmung des Bikarbonatgehaltes mit Säure kann dahingegen sehr genau ausgeführt werden, auch in stark verdünnten Lösungen.

Weiter habe ich geprüft, ob man freie Lauge resp. Bikarbonat neben Karbonat auch konduktometrisch bestimmen kann. Zwar kann man diese Stoffe mit Hilfe von Farbeindikatoren einfach bestimmen; die konduktometrische Untersuchung ist hier aber wieder von Interesse in bezug auf farbige Flüssigkeiten.

Z. anorg. u. allg. Chem, Bd. 112. 
Von den verschiedenen Untersuchungen, welche ich angestellt habe, werde ich nur einige erwähnen. Wenn man freie Lauge in Natriumkarbonat durch Titration mit Salzsäure bestimmen will, dann wird man bei der Neutralisation zuerst eine Verminderung der Leitfähigkeit finden, bis die freie Lauge neutralisiert ist: danach wird die Leitfähigkeit bei weiterer Zufügung von Säure sich wenig mehr ändern, bis das Karbonat in Bikarbonat verwandelt ist. Sehr kleine Mengen freier Lauge kann man aber nicht mehr scharf bestimmen, was auch zu erwarten ist, da die Hydrolyse des Natriumkarbonats nun sehr störend wirkt. Die Titration des Gehalts an beizender Lauge nach WINKLER gibt in diesem Falle viel bessere Resultate.

Wenn man Bikarbonat in Karbonat durch konduktometrische Titration mit Lauge bestimmen will, wird die Leitfähigkeit bei der Zufügung von Lauge zunehmen, um nach der Neutralisation zu Karbonat stärker zuzunehmen. Durch die störende Hydrolyse kann man auch hier wieder kleine Mengen Bikarbonat nicht genau bestimmen. Hierunter gebe ich einige Beispiele, welche das Gesagte näher erläutern.

\section{$25 \mathrm{ccm} 0,1 \mathrm{n}-\mathrm{Na}_{2} \mathrm{CO}_{3}$ und $10 \mathrm{ccm} 0,098 \mathrm{n}-\mathrm{NaOH}$ mit $1 \mathrm{n}-\mathrm{HCl}$}

(Fig. 4, Kurve I).

\begin{tabular}{c|c}
\hline Zugefügte ccm Reagens & $\frac{1000-a}{a}$ \\
\hline 0 & $a, 23,05$ \\
0,2 & 21,65 \\
0,4 & 20,30 \\
0,6 & 18,95 \\
0,8 & 17,70 \\
1,0 & 16,78 \\
1,2 & 16,70 \\
1,4 & 16,78 \\
1,6 & 16,89 \\
1,8 & 17,00 \\
2,0 & 17,04 \\
2,2 & 17,27 \\
2,4 & 17,55
\end{tabular}

Erster Knickpunkt: 0,965 ccm (ber. 0,98 ccm).

In folgenden zwei Tabellen sind die Resultate von Titrationen von Bikarbonat in Karbonat mit Lauge angegeben.

Im letzten Falle lag eine Mischung von $23 \mathrm{ccm} 0,1 \mathrm{n}$-Natriumkarbonat und $1 \mathrm{ccm} 0,1 \mathrm{n}$-Bikarbonat vor. Trotz des großen Überschusses an Karbonat ergab die Titration gute Resultate.

Auch wurden unter anderen noch titriert eine Mischung $10 \mathrm{~cm}$ 
Konduktometrische Bestimmung von Kohlensäure u. ihren Salzen. IV. 163

$25 \mathrm{ccm} 0,1 \mathrm{n}-\mathrm{Na}_{2} \mathrm{CO}_{3}$ und $5 \mathrm{ccm}$ $0,1 \mathrm{n} \cdot \mathrm{HCl}$ titriert mit $0,56 \mathrm{n} \cdot \mathrm{NaOH}$ (Fig. 4, Kurve II).

\begin{tabular}{c|c}
\hline $\begin{array}{c}\text { Zugefügte } \\
\text { ccm Reagens }\end{array}$ & $\frac{1000-a}{a-}$ \\
\hline \hline 0 & 14,45 \\
0,2 & 14,94 \\
0,4 & 15,44 \\
0,6 & 16,01 \\
0,8 & 16,60 \\
1,0 & 17,50 \\
1,2 & 18,76 \\
1,4 & 20,08 \\
1,6 & 21,45 \\
1,8 & 22,78 \\
2,0 & 24,20 \\
2,2 & 25,40
\end{tabular}

Knickpunkt:

$0,90 \mathrm{~cm}=5,04 \mathrm{ccm} 0,1$ n. (ber. 5,00 ).
$25 \mathrm{ccm} \quad 0,1 \mathrm{n}-\mathrm{Na}_{2} \mathrm{CO}_{3}$ und $1 \mathrm{ccm}$ $0,1 \mathrm{n}-\mathrm{HCl}$ titriert mit $0,1 \mathrm{n} \cdot \mathrm{NaOH}$ (Fig. 4, Kurve III).

\begin{tabular}{c|c}
\hline $\begin{array}{c}\text { Zugefügte } \\
\text { ccm Reagens }\end{array}$ & $\frac{1000-a}{a}$ \\
\hline \hline 0 & 16,46 \\
0,2 & 17,97 \\
0,4 & 19,50 \\
0,6 & 21,05 \\
0,8 & 22,67 \\
1,0 & 24,60 \\
1,2 & 26,90 \\
1,4 & 29,84 \\
1,6 & 32,73 \\
1,8 & 35,75 \\
2,0 & 38,5 \\
2,2 & 41,5
\end{tabular}

Knickpunkt:

$1,00 \mathrm{ccm}$ (ber. $1,00 \mathrm{ccm}$ ).

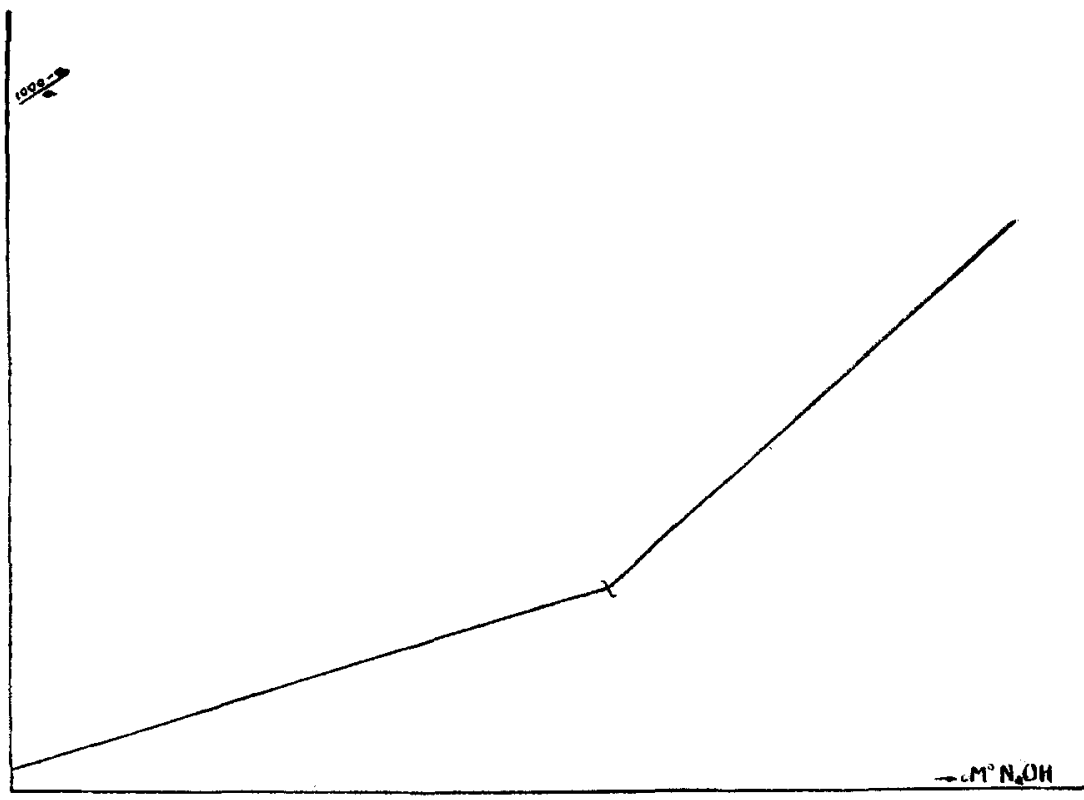

Fig. 5.

0,1 n-Bikarbonat und $5 \mathrm{cem}$ 0,1 n-Karbonat. Diese Mischung band $1,05 \mathrm{ccm}$ n-Lauge, während die berechnete Zahl $1,00 \mathrm{ccm}$ ist.

Weiter wurden $25 \mathrm{ccm}$ 0,1 n-Bikarbonat titriert. Die Lösung nahm 2,40-2,49 ccm n-Lauge (ber. $2,50 \mathrm{ccm}$ ).

Die Resultate sind also sehr befriedigend. 
$164 J . M$. Kolthoff. Konduktometr. Bestimmung von Kohlensäure usw: IV.

SchlieBlich wurde untersucht, ob man eine sehr schwache Säure auch neben Natriumkarbonat konduktometrisch bestimmen kann. Es stellte sich heraus, daß dies in der Tat der Fall war. Als Beispiel sei die Titration von Borsäure neben Natriumkarbonat gegeben, wobei man konduktometrisch ausgezeichnete Resultate erhält, während die Bestimmung mit Hilfe von einem oder mehreren Farbeindikatoren nicht ausführbar ist.

$25 \mathrm{ccm} 0,1 \mathrm{n}$-Natriumkarbonat und $25 \mathrm{ccm} 0,1 \mathrm{n}$-Borsëure mit $1,035 \mathrm{n}-\mathrm{NaOH}$ (Fig. 5).

\begin{tabular}{c|c}
\hline Zugefügte ccm Reagens & $\frac{1000-a}{u}$ \\
\hline \hline 0 & 0,4 \\
0,4 & 10,04 \\
0,8 & 11,25 \\
1,2 & 12,45 \\
1,6 & 13,70 \\
2,0 & 14,69 \\
2,4 & 16,46 \\
2,8 & 19,67 \\
3,0 & 21,50 \\
3,2 & 23,30 \\
3,4 & 25,1 \\
3,8 & 28,7
\end{tabular}

(Knickpunkt: $2,42 \mathrm{ccm}=2,505 \mathrm{~cm} \mathrm{n}$. (ber. $2,50 \mathrm{ccm}$ ).

\section{Zusammenfassung der Ergeb nisse.}

1. Die konduktometrische Titration von Kohlensäure ergibt nicht sehr genaue Resultate. Die Knickpunkte bei Karbonat und Bikarbonat sind zu unscharf. Verbesserung kann durch Hinzufügung eines Überschusses an Calciumsalz gemacht werden. Man soll dem Calciumkarbonat aber während der Titration Zeit lassen, zu präzipitieren.

2. Karbonat kann nicht scharf zu Bikarbonat, wohl aber zu Kohlensäure titriert werden. Auch in äußerst verdünnten Lösungèn ergibt diese konduktometrische Titration noch gute Resultate.

3. Freie Lauge oder Bikarbonat können neben Karbonat genau bestimmt werden, wenn das Verhältnis der Konzentrationen der Stoffe nicht zu ungünstig ist.

Utrecht, Pharmazeut. Laboratorium der Universität. Januar 1920.

Bei der Redaktion eingegangen am 30. April 1920. 